\title{
Kalai's squeezed 3-spheres are polytopal
}

\author{
JULiAn PfEifle* \\ Dept. of Mathematics, MA 6-2 \\ TU Berlin, D-10623 Berlin, Germany \\ pfeifle@math.tu-berlin.de
}

October 19, 2001

\begin{abstract}
In 1988, KALAI [5] extended a construction of BILLERA and LEE to produce many triangulated $(d-1)$-spheres. In fact, in view of upper bounds on the number of simplicial $d$-polytopes by Goodman and Pollack [2, 3], he derived that for every dimension $d \geq 5$, most of these $(d-1)$-spheres are not polytopal. However, for $d=4$, this reasoning fails. We can now show that, as already conjectured by KALAI, all of his 3 -spheres are in fact polytopal.

We also give a shorter proof for HEBBLE and LEE's result [4] that the dual graphs of these 4-polytopes are Hamiltonian.
\end{abstract}

\section{Introduction}

This paper is about triangulated spheres and the question whether or not the members of a certain family of them are realizable, that is, if they arise as boundary complexes of simplicial polytopes. While for all two-dimensional spheres this is true by Steinitz' Theorem, already one dimension higher there exist simplicial spheres that cannot be realized in a convex way. The first example for this - the so-called Brückner sphere - was found by GrünBAUM \& SREEDHARAN in 1967, who realized that a certain simplicial 3 -sphere on 8 vertices does not represent the combinatorial type of any 4-polytope, contrary to what BRÜCKNER originally thought. (See [11, Chapter 5] for a more thorough discussion and references.)

In 1988, KALAI extended a construction by BILLERA and LEE, and showed that starting with $d=5$, there exist many more simplicial $(d-1)$-spheres than simplicial $d$-polytopes, and that therefore, in a very strong sense, most simplicial spheres are not realizable. In contrast, it is the main goal of this paper to show that all of KALAI's 3-spheres do arise as boundary complexes of simplicial 4-polytopes.

In the remainder of this introduction, we present the context of these constructions, including the known upper resp. lower bounds for the numbers of simplicial polytopes resp. spheres.

The most important invariant of a $(d-1)$-dimensional simplicial sphere $\mathcal{S}$ is its f-vector $f(\mathcal{S})=\left(f_{-1}, f_{0}, f_{1}, \ldots, f_{d-1}\right)$, where $f_{i}=f_{i}(\mathcal{S})$ counts the number of $i$-dimensional faces of $\mathcal{S}$, and $f_{-1}=1$. In 1971, MCMULLEN [7] conjectured a characterization of the $f$-vectors of boundary complexes of simplicial $d$-polytopes in terms of an encoding of $f(\mathcal{S})$, the so-called $g$-vector. First define the h-vector $h(\mathcal{S})=\left(h_{0}, h_{1}, \ldots, h_{d}\right)$ of $\mathcal{S}$ by

$$
h_{k}=\sum_{i=0}^{k}(-1)^{k-i}\left(\begin{array}{l}
d-i \\
d-k
\end{array}\right) f_{i-1}, \quad \text { for } k=0,1, \ldots, d .
$$

*Graduate student at the European graduate school 'Combinatorics, Geometry, and Computation', supported by the Deutsche Forschungsgemeinschaft, grant GRK 588/1 
The $h$-vector of any simplicial sphere satisfies the Dehn-Sommerville equations $h_{k}=h_{d-k}$ for $k=0,1, \ldots,\lfloor d / 2\rfloor$. Now the g-vector of $\mathcal{S}$ is $g(\mathcal{S})=\left(g_{0}, g_{1}, \ldots, g_{\lfloor d / 2\rfloor}\right)$, where $g_{0}:=h_{0}=1$ and

$$
g_{k}:=h_{k}-h_{k-1} \quad \text { for } \quad k=1,2, \ldots,\lfloor d / 2\rfloor .
$$

We say that $g(\mathcal{S})$ forms an M-sequence if $g_{0}=1$ and $g_{k-1} \geq \partial^{k}\left(g_{k}\right)$ for $k=1, \ldots,\lfloor d / 2\rfloor$, where

$$
\partial^{k}\left(g_{k}\right)=\left(\begin{array}{c}
a_{k}-1 \\
k-1
\end{array}\right)+\left(\begin{array}{c}
a_{k-1}-1 \\
k-2
\end{array}\right)+\cdots+\left(\begin{array}{c}
a_{2}-1 \\
1
\end{array}\right)+\left(\begin{array}{c}
a_{1}-1 \\
0
\end{array}\right),
$$

and the integers $a_{k}>a_{k-1}>\cdots>a_{2}>a_{1} \geq 0$ are determined by the binomial expansion

$$
g_{k}-1=\left(\begin{array}{c}
a_{k} \\
k
\end{array}\right)+\left(\begin{array}{c}
a_{k-1} \\
k-1
\end{array}\right)+\cdots+\left(\begin{array}{c}
a_{2} \\
2
\end{array}\right)+\left(\begin{array}{c}
a_{1} \\
1
\end{array}\right)
$$

of $g_{k}-1$ w.r.t. $k$. See [11, Chapter 8] for more details. We can now state McMulLEN's conjecture:

Theorem 1. (g-conjecture/theorem) An integer vector $g=\left(g_{0}, g_{1}, \ldots, g_{\lfloor d / 2\rfloor}\right)$ is the g-vector of the boundary complex of a simplicial d-polytope $P$ if and only if it is an M-sequence.

In the same year, 1979, StANlEy 9] proved the necessity and Billera and LeE [1] the sufficiency of MCMULLEN's conditions. STANLEY's proof that the $g$-vector of any simplicial polytope is an M-sequence used the Hard Lefschetz Theorem for the cohomology of projective toric varieties, but in the meantime a simpler proof by MCMULLEN using his polytope algebra is available.

BILlERA and LEE invented an ingenious construction to produce, for every M-sequence $g$, a simplicial $d$-polytope with this $g$-vector. Very briefly, they first find a shellable ball $B$ as a collection of facets of a cyclic polytope $C$, such that the $g$-vector of $\partial B$ is the given M-sequence. Then they construct a realization of $C$ and a point $z$ that sees exactly the facets in $B$, and obtain a realization of $\partial B$ as a simplicial polytope by taking the vertex figure at $z$ of $\operatorname{conv}(\{z\} \cup C)$.

We next discuss KaLAI's 1988 extension of their construction, by which he built so many simplicial spheres that most of them (in a sense to be made precise below) fail to be polytopal. He achieved this by giving a rule to produce many lists $I$ of $(d+1)$-tuples of vertices, which span pure simplicial complexes $B(I)$. The underlying space of every such complex turns out to be a simplicial, shellable $d$-ball, which he called a squeezed ball, and therefore the boundary $S(I)$ of $B(I)$ is a simplicial $(d-1)$-sphere, a squeezed sphere. LEE shows in [6] that KaLAI's squeezed spheres are shellable.

Let $s(d, n)$ denote the number of simplicial $(d-1)$-spheres, $s q(d, n)$ the number of squeezed $(d-1)$-spheres, and $c(d, n)$ the number of combinatorial types of simplicial $d$-polytopes with $n$ labeled vertices. Goodman and Pollack [2, 3] derive the upper bound

$$
\log c(d, n) \leq d(d+1) n \log n
$$

using a theorem of MiLnOR that bounds the sum of the Betti numbers of real algebraic varieties, while KALAI's squeezed spheres provide the following lower bound for $s(d, n)$ :

$$
\begin{aligned}
\log s(d, n) \geq \log s q(d, n) & \geq \frac{1}{(n-d)(d+1)}\left(\begin{array}{c}
n-\lfloor(d+2) / 2\rfloor \\
\lfloor(d+1) / 2\rfloor
\end{array}\right) \\
& =\Omega\left(n^{\lfloor(d+1) / 2\rfloor-1}\right) \quad \text { for fixed } d .
\end{aligned}
$$

These bounds reveal that $\lim _{n \rightarrow \infty} c(d, n) / s q(d, n)=0$ for $d \geq 5$, which means that for $d \geq 5$ most of KALAI's spheres are not polytopal - there are simply too many of them. However, we learn nothing for $d \leq 4$ : We will prove in Proposition 1 below that $s q(4, n) \leq 2^{n-5} n$ ! for $n \geq 5$, which is strictly less than the bound from (11) for all $n \geq 5$.

The rest of the paper is organized as follows: In Section 2, we collect some facts about cyclic polytopes, an essential ingredient of our proof. In Section 3, we first present the details of KALAI's construction, and then show how to realize any of his 3 -spheres as boundary complexes of simplicial 4-polytopes (Theorem 2). Finally, Section 4 uses the pictures constructed in Section 3 to give a shorter proof of HEBBLE and LEE's result that the dual graphs of squeezed 3-spheres are Hamiltonian. 


\section{Some facts on cyclic polytopes}

The convex hull of $n$ distinct points on the moment curve $\mu_{d}: t \mapsto\left(t, t^{2}, \ldots, t^{d}\right)$ in $\mathbb{R}^{d}$ is called a $d$-dimensional cyclic polytope with $n$ vertices. The combinatorial type of this polytope is independent of the choice of the $n$ points on the moment curve, and so one can talk about the cyclic polytope $C_{d}(n)$. In fact, any $d$-dimensional order $d$ curve also gives rise to the same combinatorial types of polytopes.

We switch from $d$ and $n$ to $d+1$ and $n+1$, and consider a set $X=\left\{x_{0}=\mu\left(t_{0}\right), \ldots, x_{n}=\mu\left(t_{n}\right)\right\}$ of $n+1$ distinct points on the moment curve $\mu_{d+1}=: \mu$, ordered by their first coordinates. For any $f \subset\{0,1, \ldots, n\}$, write $F_{f}$ for the subset of $X$ indexed by $f$, and $i(F)$ for the indices of a subset $F$ of $X$. The supporting hyperplane $H(F)$ of a $(d+1)$-subset $F \subset X$ is given by $H(F)=\left\{x \in \mathbb{R}^{d+1}: \gamma(F) \cdot x=-\gamma_{0}(F)\right\}$, where $\gamma(F)=\left(\gamma_{1}(F), \ldots, \gamma_{d+1}(F)\right) \in \mathbb{R}^{d+1}$ and $\gamma_{0}(F) \in \mathbb{R}$ are defined by

$$
0=\prod_{i \in i(F)}\left(t-t_{i}\right)=\sum_{j=0}^{d+1} \gamma_{j}(F) t^{j}=\gamma_{0}(F)+\gamma(F) \cdot \mu(t) .
$$

Observe that $\gamma_{d+1}(F)=1$; we say that $\gamma(F)$ points upwards.

GALE's evenness criterion tells us which $(d+1)$-subsets $F$ of $X$ are vertex sets of facets of the cyclic polytope $C=\operatorname{conv}(X)$ : For any $i, j \in\{0,1, \ldots, n\} \backslash i(F)$, the number of elements of $i(F)$ between $i$ and $j$ must be even.

Define the end set $W_{\text {end }}$ of $F_{f} \subset X$ to be the right-most contiguous block $\left\{r_{f}+1, \ldots, \max f\right\}$ of the indices $f$ of $F$, where $r_{f}=\max \{i \in \mathbb{N}: i<\max f, i \notin f\}$. Let $F$ be a facet of $C$ and take $x_{j}=\mu\left(t_{j}\right) \in X \backslash F$. If the cardinality of the end set of $F$ is odd, we get $\prod_{i \in i(F)}\left(t_{j}-t_{i}\right)<0$ because $j \notin i(F)$, and therefore $\gamma(F) \cdot x_{j}<-\gamma_{0}(F)$. Since $\gamma_{d+1}(F)=1$, we conclude that the whole cyclic polytope $C$ is below $F$, and call $F$ an upper facet of $C$. If $\# W_{\text {end }}$ is even, we analogously call $F$ a lower facet of $C$. Finally, define an outer normal vector $\alpha(F)$ of any facet $F$ of $C$ by $\alpha(F)=\gamma(F)$ resp. $\alpha(F)=-\gamma(F)$ if $F$ is an upper resp. lower facet of $C$, and set $\alpha_{0}(F)=-\gamma_{0}(F)$ resp. $\alpha_{0}(F)=\gamma_{0}(F)$. By this, we obtain $C \subset\left\{x \in \mathbb{R}^{d+1}: \alpha(F) \cdot x \leq \alpha_{0}(F)\right\}$ for all facets $F$ of $C$.

\section{Realizing Kalai's 3-spheres}

\subsection{Kalai's idea}

First define a partial order $\preceq$ on $\left(\begin{array}{c}\mathbb{N} \\ d+1\end{array}\right)$ by $\left\{i_{1}, i_{2}, \ldots, i_{d+1}\right\}_{<} \preceq\left\{j_{1}, j_{2}, \ldots, j_{d+1}\right\}_{<}$if $i_{k} \leq j_{k}$ for every $k=1, \ldots, d+1$. Here the notation $A=\left\{a_{1}, \ldots, a_{r}\right\}<$ means that the elements of the set $A$ are listed in increasing order. For the standard poset terminology used in the following, see [10].

For an odd integer $d>0$ and $n \in \mathbb{N}$, let $\mathcal{F}_{d}(n)$ be the collection of $(d+1)$-subsets of $[n]:=$ $\{1,2, \ldots, n\}$ of the form $\left\{i_{1}, i_{1}+1\right\} \cup\left\{i_{2}, i_{2}+1\right\} \cup \cdots \cup\left\{i_{e}, i_{e}+1\right\}$, where $e=(d+1) / 2, i_{1} \geq 1$, $i_{e}<n$, and $i_{j+1} \geq i_{j}+2$ for all relevant $j$. Let $I^{\prime}$ be an initial set (order ideal) of $\mathcal{F}_{d}(n)$ with respect to the partial order $\preceq$ on $\left(\begin{array}{c}\mathbb{N} \\ d+1\end{array}\right)$. Informally, $f^{\prime} \preceq g^{\prime}$ for $f^{\prime}, g^{\prime} \in \mathcal{F}_{d}(n)$ if $f^{\prime}$ arises from $g^{\prime}$ by pushing some elements in $g^{\prime}$ to the left.

For even $d>0$, put $\mathcal{F}_{d}(n)=\left\{\{0\} \cup f^{\prime}: f^{\prime} \in \mathcal{F}_{d-1}(n)\right\}=: 0 * \mathcal{F}_{d-1}(n)$ with the induced partial order, and set $I:=0 * I^{\prime}$.

Finally, let $B(I)$ be the simplicial complex (the squeezed $d$-ball) spanned by $I$, denote the boundary complex of $B(I)$ by $S(I)$ (the squeezed $(d-1)$-sphere), and do the same for $I^{\prime}$.

\subsection{The structure of 3-balls}

To specialize KaLAI's construction to $d=4$, we first study squeezed 3-balls. Take $n \geq 4$ in $\mathbb{N}$, write $(i, j)$ for an element $\{i, i+1, j, j+1\} \subset[n]$ of $\mathcal{F}_{3}(n)$, and define the gap of $(i, j) \in \mathcal{F}_{3}(n)$ 
to be the number $j-i-2$ of integers between $i+1$ and $j$. From the fact that any two elements of $\mathcal{F}_{3}(n)$ with the same gap are translates of each other and therefore $\preceq$-comparable, we conclude that any $\preceq$-antichain in $\mathcal{F}_{3}(n)$ can be linearly ordered by increasing gap, and denote this order by $\sqsubset$. We remark that the difference between the gaps of any two elements in a $\preceq$-antichain must be at least 2 , as otherwise the two elements would be $\preceq$-comparable. In particular, the maximal number of elements of a $\preceq$-antichain in $\mathcal{F}_{3}(n)$ is $\lceil(n-3) / 2\rceil$.

Any order ideal $I^{\prime} \subset \mathcal{F}_{3}(n)$ for $n \in \mathbb{N}$ is generated by the set $G^{\prime}=\left\{g_{1}^{\prime}, g_{2}^{\prime}, \ldots, g_{r}^{\prime}\right\}_{\sqsubset}$ of its maximal elements, for some $r \leq\lceil(n-3) / 2\rceil$. By our discussion, the $g_{k}^{\prime}=\left(i_{k}, j_{k}\right)$ satisfy

(1) $j_{k} \geq i_{k}+2$ for $k=1, \ldots, r$, and

(2) $i_{k}>i_{k+1}$ and $j_{k}<j_{k+1}$ for $k=1, \ldots, r-1$.

As an example, let $I^{\prime}$ be the ideal generated by $G^{\prime}=\{(9,11),(8,12),(5,14),(2,17)\}_{\sqsubset}$ :

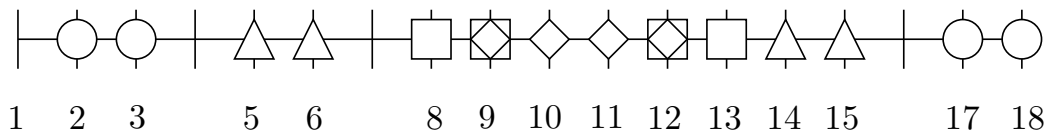

Note that if $g^{\prime} \sqsubset h^{\prime} \in G^{\prime}$, then $g^{\prime}$ is nested inside $h^{\prime}$ (possibly with overlap). From Figure 1 below, we will read off the structure of the 3-ball $B\left(I^{\prime}\right)$ generated by $G^{\prime}$, and its boundary $S\left(I^{\prime}\right)$.

Now put $\mathcal{F}_{4}(n)=0 * \mathcal{F}_{3}(n)$ with the induced partial order, and $I=0 * I^{\prime}$. The 4-ball $B(I)$ spanned by $I$ is a cone over the 3 -ball $B\left(I^{\prime}\right)$, whose boundary complex is the squeezed 3 -sphere $S(I)$.

Proposition 1. There are at most $2^{n-4}(n+1)$ ! squeezed 3 -spheres with $n+1 \geq 5$ labeled vertices. In particular, $\log s q(4, n)=\Theta(n \log n)$.

Proof. By [5, Prop. 3.3], distinct 4-balls $B(I)$ whose vertices are labeled according to their construction give rise to distinct 3-spheres $S(I)$ labeled in this way, and distinct initial sets $I \subset \mathcal{F}_{4}(n)$ obviously induce distinct such 4-balls. Every initial set $I$ is of the form $0 * I^{\prime}$ for a unique order ideal $I^{\prime} \subset \mathcal{F}_{3}(n)$. Therefore, by relabeling vertices, $s q(4, n+1)$ is at most $(n+1)$ ! times the number of distinct order ideals in $\mathcal{F}_{3}(n)$, depending on the combinatorial symmetries of $S(I)$. By Figure 1, every such order ideal can be represented by a lattice path of length $n-4$ taking steps only in the positive $i$ - or negative $j$-directions, and starting at $(i, j)=(1, n-1)$. There are $2^{n-4}$ of these, and they all give rise to distinct ideals.

\subsection{A bird's-eye view of the realization construction}

Observe that by GALE's Evenness Criterion, every $f \in I$ corresponds to a lower facet $F_{f}$ of a cyclic polytope. By adapting the ideas of BILlerA and LEE, we will now realize any $S(I)$ as the boundary complex of a 4-polytope $P$ by appropriately realizing a cyclic 5-polytope $C$, and choosing a viewpoint $v$ close to the negative $e_{5}$-axis that sees exactly the facets of $C$ in $B(I)$. The convex 4-polytope $P$ is then the vertex figure at $v$ of $\operatorname{conv}(C \cup\{v\})$, and $S(I)$ its boundary.

Specifically, let $\mu=\mu_{5}: \mathbb{R} \rightarrow \mathbb{R}^{5}, t \mapsto\left(t, t^{2}, \ldots, t^{5}\right)$ be the moment curve in dimension 5 . Given an order ideal $I=0 * I^{\prime}$ in $\mathcal{F}_{4}(n)$ where $n=\max \bigcup I$, we will execute the following steps:

1. Choose $N^{\prime}>0$ and place $0=t_{0}<t_{1}<\cdots<t_{n} \in \mathbb{R}_{\geq 0}$ such that

$$
\begin{aligned}
& \prod_{i \in f \backslash\{0\}} t_{i}<N^{\prime} \quad \text { for all } f \in I, \quad \text { and } \\
& \prod_{i \in f \backslash\{0\}} t_{i}>N^{\prime} \quad \text { for all } f \in \mathcal{F}_{4}(n) \backslash I
\end{aligned}
$$

Solutions for (S1) exist with $t_{1}>0$ arbitrarily small. We will find a solution for this system of inequalities by processing the elements of $E^{\prime}=G^{\prime} \cup H^{\prime}$ in ᄃ-order, where $G^{\prime}$ is the set of $\preceq$-maximal elements of $I^{\prime}$, and $H^{\prime}$ is the set of $\preceq$-minimal elements of $\mathcal{F}_{3}(n) \backslash I^{\prime}$. 


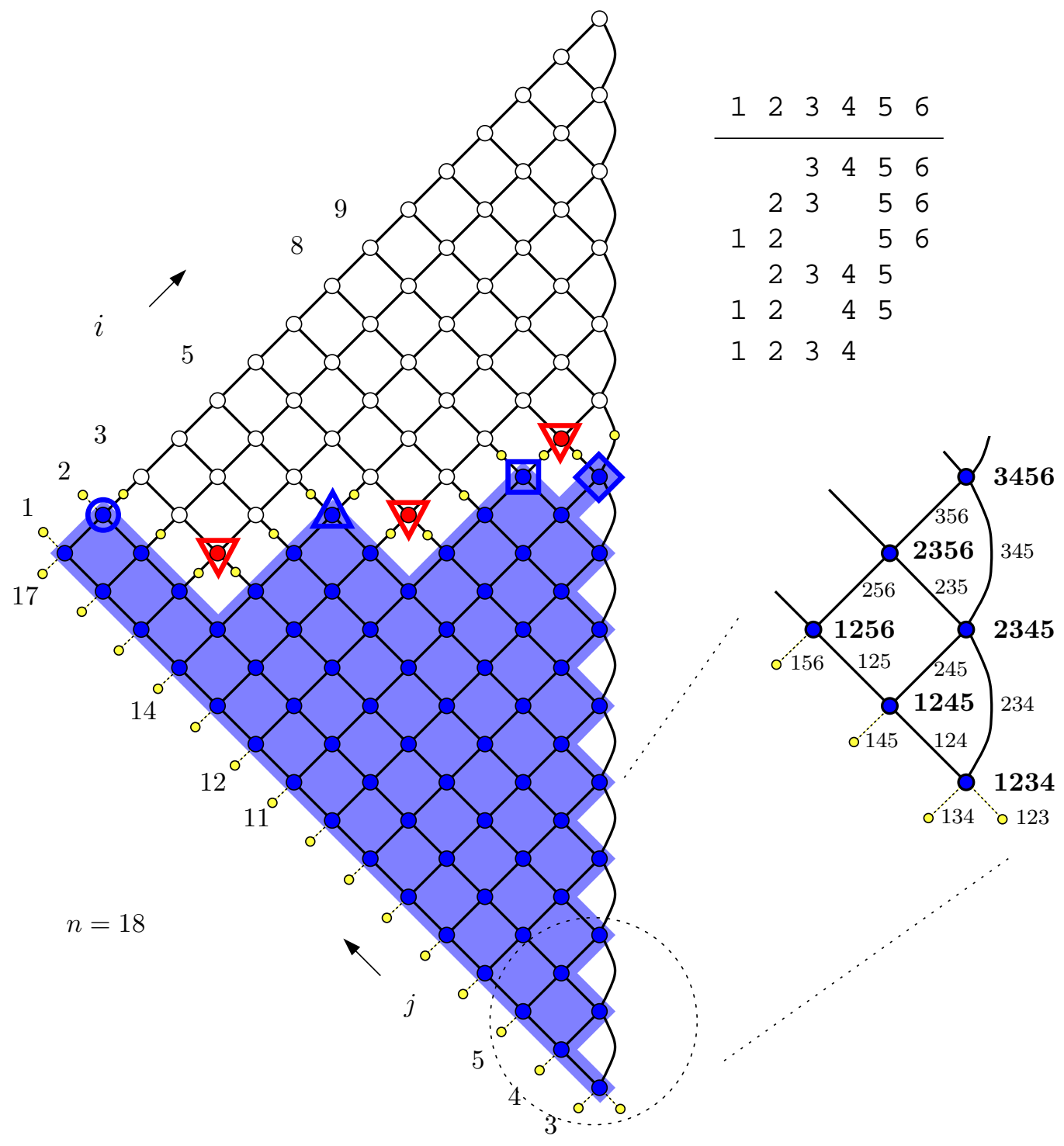

Figure 1: The Kalai poset $\mathcal{F}_{3}(18)$. The shaded circles are the facets of the 3 -ball $B\left(I^{\prime}\right)$ with generators $G^{\prime}=\{(9,11),(8,12),(5,14),(2,17)\}_{\sqsubset}$. The minimal elements $H^{\prime}$ of $\mathcal{F}_{3}(18) \backslash I^{\prime}$ are marked by $\nabla$ 's, and $\sqsubset$ orders the elements of $E^{\prime}=G^{\prime} \cup H^{\prime}$ from left to right (see Observation 1 i). Straight lines between facets correspond to $\prec$-covering relations between elements of $I^{\prime}$, and straight and curved lines together to inner ridges of $B\left(I^{\prime}\right)$. The small circles are the facets of $S\left(I^{\prime}\right)=\partial B\left(I^{\prime}\right)$. The set of facets of the Kalai sphere $S(I)$ is the union of $B\left(I^{\prime}\right)$ and $0 * S\left(I^{\prime}\right)$. 
2. Make sure that the viewpoint to be defined will not see any upper facets of $C=C_{5}(n+1)=$ $\operatorname{conv}\left\{0, \mu\left(t_{1}\right), \mu\left(t_{2}\right), \ldots, \mu\left(t_{n}\right)\right\}$ that contain 0 , by choosing $t_{1}>0$ so small that

$$
t_{1} t_{n-2} t_{n-1} t_{n}<N^{\prime}
$$

3. Choose $\varepsilon$, with $0<\varepsilon<t_{1}$, so small that for all $e, f \in \mathcal{F}_{4}(n)$,

$$
e \prec f \quad \Longrightarrow \quad \gamma\left(F_{e}\right) \cdot \mu(\varepsilon)<\gamma\left(F_{f}\right) \cdot \mu(\varepsilon) .
$$

4. Choose $\varepsilon>0$ even smaller, if necessary, such that the viewpoint $v:=\mu(\varepsilon)-\varepsilon N^{\prime} e_{5}$ satisfies

$$
\begin{array}{ll}
\alpha(F) \cdot v>\alpha_{0}(F) & \text { for } f_{F} \in I, \\
\alpha(F) \cdot v<\alpha_{0}(F) & \text { for all lower facets } F \text { of } C \text { such that } f_{F} \notin I, \\
\alpha(F) \cdot v<\alpha_{0}(F) & \text { for all upper facets } F \text { of } C,
\end{array}
$$

where $\alpha(F)$ is the outer normal vector of $F$ we defined at the end of Section 2.

We conclude that $v$ sees exactly the facets of $C$ in $B(I)$, and obtain $S(I)$ as above.

\subsection{How to realize Kalai's 3-spheres}

We will now give the details of the construction and prove the following theorem.

Theorem 2. Every squeezed 3 -sphere $S(I)$ given by an order ideal $I$ in the poset $\left(\mathcal{F}_{4}(n), \preceq\right)$ with $n \geq \max \bigcup I$ can be realized as the boundary complex of a simplicial, convex 4-polytope.

Remark 1. The construction shows the stronger result that every squeezed 4-ball $B(I)$ can be realized as a regular triangulation of a convex 4-polytope.

To prove Theorem 2, given an ideal $I \subset \mathcal{F}_{4}(n)$, we may assume that $n=\max \bigcup I$ since $\mathcal{F}_{4}(n) \subseteq \mathcal{F}_{4}\left(n^{\prime}\right)$ for $n \leq n^{\prime}$. By definition, every order ideal $I \subset \mathcal{F}_{4}(n)$ has the form $I=0 * I^{\prime}$, where $I^{\prime}=\left\langle G^{\prime}\right\rangle \subset \mathcal{F}_{3}(n)$ is generated by its maximal elements $G^{\prime}=\left\{g_{1}^{\prime}, g_{2}^{\prime}, \ldots, g_{r}^{\prime}\right\}$ with $g_{k}^{\prime}=\left(i_{k}, j_{k}\right)$. Choose $N^{\prime}>0$, introduce $n$ variable points $0<t_{1}<t_{2}<\cdots<t_{n}$ in $\mathbb{R}_{>0}$, and consider the set $H^{\prime}$ of $\preceq$-minimal elements of $\mathcal{F}_{3}(n) \backslash I^{\prime}$.

Observation 1. Consider any two consecutive elements $e^{\prime}=(i, j) \sqsubset f^{\prime}=(k, \ell)$ of a $\sqsubset$-ordered $\preceq$-antichain $G^{\prime}$ of $\mathcal{F}_{3}(n)$. Then the unique $\prec$-minimal element $m^{\prime}$ in $\mathcal{F}_{3}(n) \backslash\left\langle G^{\prime}\right\rangle$ with $\operatorname{gap}\left(e^{\prime}\right)<$ $\operatorname{gap}\left(m^{\prime}\right)<\operatorname{gap}\left(f^{\prime}\right)$ exists and is $m^{\prime}=(k+1, j+1)$. In particular, the number of $\prec$-minimal elements in $\mathcal{F}_{3}(n) \backslash\left\langle G^{\prime}\right\rangle$ is no greater than $\lfloor(n-3) / 2\rfloor$.

Sketch of proof. The first statement follows by inspection of Figure 1. For the second assertion, note that the set $H^{\prime}$ has maximal cardinality if $G^{\prime}=\{(i, n-i): i=1,2, \ldots,\lceil(n-3) / 2\rceil\}$.

Using Observation 1, we linearly order $E^{\prime}=G^{\prime} \cup H^{\prime}$ by $\sqsubset$, see Figure 1. To carry out Step 1 of our program, first choose some small $\delta>0$. Our goal is to place the $t$ 's in $\mathbb{R}_{>0}$ such that

$$
\prod_{i \in g^{\prime}} t_{i}=N^{\prime}-\delta \quad \text { for } g^{\prime} \in G^{\prime} \quad \text { and } \quad \prod_{i \in h^{\prime}} t_{i}=N^{\prime}+\delta \quad \text { for } h^{\prime} \in H^{\prime} .
$$

Observation 2. The cardinality of $E^{\prime}=G^{\prime} \cup H^{\prime}$ is at most $n-3$. In particular, there are fewer equalities in (S1) than there are variables. 
Proof. Because $n=\max \bigcup I$, the largest element of $\left(E^{\prime}, \sqsubset\right)$ is in $G^{\prime}$. Using Observation 1 again,

$$
\# E^{\prime}=\# G^{\prime}+\# H^{\prime} \leq\left\lceil\frac{n-3}{2}\right\rceil+\left\lfloor\frac{n-3}{2}\right\rfloor=n-3,
$$

which proves Observation 2 .

We now begin the construction by placing the t's corresponding to the $\sqsubset$-smallest element of $E^{\prime}$ in such a way in $\mathbb{R}_{>0}$ that $(\overline{\mathrm{S} 1})$ is satisfied. This is clearly possible. The general step of constructing a solution to $(\overline{\mathrm{S} 1})$ is based on the following lemma.

Lemma 1. Let $e^{\prime}=(i, j) \sqsubset f^{\prime}=(k, \ell)$ be two consecutive elements of $E^{\prime}$.

(a) If $e^{\prime} \in G^{\prime}$ and $f^{\prime} \in H^{\prime}$, then $0<k \leq i$ and $\ell=j+1$. If $e^{\prime} \in H^{\prime}$ and $f^{\prime} \in G^{\prime}$, then $k=i-1$ and $j \leq \ell<n$. (See Figure 团.)

(b) Suppose that the $\left\{t_{i}\right\}_{i \in e^{\prime}}$ have been placed already, but not all $\left\{t_{j}\right\}_{j \in f^{\prime}}$. Then these latter $t^{\prime} s$ may be placed in such a way in $\mathbb{R}_{>0}$ that $0<t_{k}<t_{k+1}<t_{\ell}<t_{\ell+1}$, and the equality

$$
t_{k} t_{k+1} t_{\ell} t_{\ell+1}=M
$$

is satisfied, where $M:=N^{\prime}-\delta$ if $f^{\prime} \in G^{\prime}$ and $M:=N^{\prime}+\delta$ if $f^{\prime} \in H^{\prime}$.

Sketch of proof for (D). Suppose that $e^{\prime} \in G^{\prime}$ and $f^{\prime} \in H^{\prime}$. We then have the following situation:

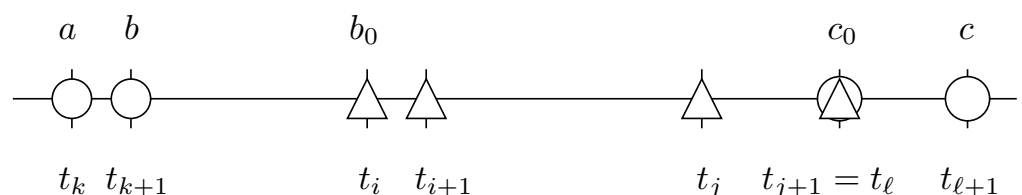

It is straightforward to verify that for any $0<k \leq i$, the points $a, b, c$ may be placed in such a way that $0<a<b<b_{0}<c_{0}<c$ and $a b c_{0} c=N^{\prime}+\delta$. Similarly, if $e^{\prime} \in H^{\prime}$ and $f^{\prime} \in G^{\prime}$,

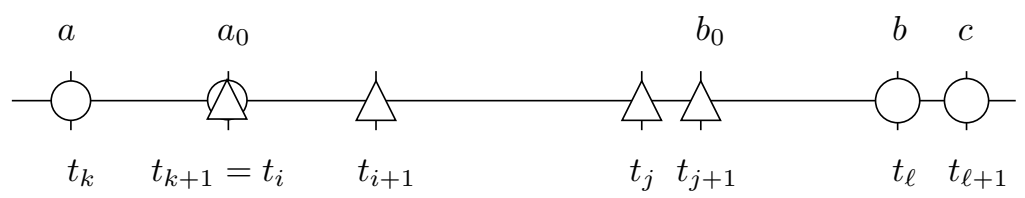

for any $j \leq \ell<n$ we may place $a, b, c$ such that $0<a<a_{0}<b_{0}<b<c$ and $a a_{0} b c=N^{\prime}-\delta$.

We now complete Step 1 by applying Lemma 1 to all members of $E^{\prime}$ in $\sqsubset$-order. The definition of $\preceq$ tells us that because the $f^{\prime} \in E^{\prime}$ satisfy (S1 ), in fact all $f \in \mathcal{F}_{4}(n)$ satisfy the system (S1).

If in Step 1 we encountered some $e^{\prime} \in E^{\prime}$ with $1 \in e^{\prime}$, then necessarily $e^{\prime}=\{1,2, n-1, n\} \in G^{\prime}$, which imposed the inequality $t_{1} t_{2} t_{n-1} t_{n}<N^{\prime}$. This inequality in turn remains satisfied if we choose $t_{1}$ even small enough to verify (S2). If $1 \notin e^{\prime}$ for all $e^{\prime} \in E^{\prime}$, we are free to do the same. We have completed Step 2, and place any remaining unassigned $t$ 's such that $0=t_{0}<t_{1}<\cdots<t_{n}$.

Observation 3. (a) $\gamma_{0}\left(F_{f}\right)=0$ for any 5 -element subset $f \subset\{0,1, \ldots, n\}$ that contains 0 .

(b) For all choices of $t_{1}<\cdots<t_{n}$, one can find $\varepsilon>0$ small enough such that the implication (S3) holds for all $f, g \in \mathcal{F}_{4}(n)$.

Proof of (B). The definition (2) of the $\gamma^{\prime}$ 's implies that for $f=\left\{0, s_{1}, \ldots, s_{4}\right\}$,

$$
\gamma\left(F_{f}\right) \cdot \mu(\varepsilon)=\varepsilon\left(\varepsilon-s_{1}\right) \cdots\left(\varepsilon-s_{4}\right)=\varepsilon s_{1} s_{2} s_{3} s_{4} \pm o(\varepsilon) .
$$

This means that $\gamma\left(F_{f}\right) \cdot \mu(\varepsilon)<\gamma\left(F_{g}\right) \cdot \mu(\varepsilon)$ by definition of $\prec$, for $\varepsilon$ small enough. 
Take $0<\varepsilon<t_{1}$ as in Observation (3(B)), tentatively set $z:=\mu(\varepsilon)$, and let $f \in \mathcal{F}_{4}(n)$. If $f \in I$, there exists some $g \in G:=0 * G^{\prime}$ with $f \preceq g$, and by (何, we have

$$
\gamma\left(F_{f}\right) \cdot z \leq \gamma\left(F_{g}\right) \cdot z=\varepsilon \prod_{i \in g \backslash\{0\}} t_{i}+O\left(\varepsilon^{2}\right)=\varepsilon\left(N^{\prime}-\delta\right) \pm o(\varepsilon) .
$$

If $f \notin I$, then there is some $h \in H:=0 * H^{\prime}$ with $f \succeq h$, and we obtain in a similar way that

$$
\gamma\left(F_{f}\right) \cdot z \geq \varepsilon\left(N^{\prime}+\delta\right) \pm o(\varepsilon) .
$$

Thus, we finally choose $0<\varepsilon<t_{1}$ so small that with $z:=\mu(\varepsilon)$ and $N:=\varepsilon N^{\prime}$, we have $\gamma\left(F_{f}\right) \cdot z<N$ for $f \in I$, and $\gamma\left(F_{f}\right) \cdot z>N$ for $f \notin I$. Step 3 is now complete.

We proceed to verify that $v:=\mu(\varepsilon)-\varepsilon N^{\prime} e_{5}=z-N e_{5}$ satisfies the inequalities (S4). For this, recall that all $F_{f}$ with $f \in \mathcal{F}_{4}(n)$ satisfy GALE's Evenness Criterion, which means that $\mathcal{F}_{4}(n)$ is exactly the set of lower facets of the cyclic polytope $C=\operatorname{conv}(X)$ that contain $x_{0}=0$. However, any $F \subset X$ of odd cardinality satisfying GALE's Evenness Criterion with even end-set must contain 0 , and we conclude that $\mathcal{F}_{4}(n)$ is in fact the set of all lower facets of $C$.

Recall from Section 2 that $\alpha(F)=\gamma(F)$ and $\alpha_{0}(F)=-\gamma_{0}(F)$ if $F$ is an upper facet of $C$, and that $\alpha(F)=-\gamma(F)$ and $\alpha_{0}(F)=\gamma_{0}(F)$ if $F$ is a lower facet of $C$. We and discuss all facets $F_{f}$ of $C$ in turn:

Lower facets of $C$ :

- If $f \in I \subset \mathcal{F}_{4}(n)$, then by construction $\gamma\left(F_{f}\right) \cdot z<N$, and this implies $\gamma\left(F_{f}\right) \cdot v<0$ (remember that $\gamma_{5}(F)=1$ for all $F$ ) and $\alpha\left(F_{f}\right) \cdot v>0=\alpha_{0}\left(F_{f}\right)$, which means that $F_{f}$ is visible from $v$.

- If $f \in \mathcal{F}_{4}(n) \backslash I$, we conclude from $\gamma\left(F_{f}\right) \cdot z>N$ that $\alpha\left(F_{f}\right) \cdot v<0=\alpha_{0}\left(F_{f}\right)$, which says that $F_{f}$ is not visible from $v$.

Upper facets of $C$ :

- If $0 \notin f=\left\{s_{1}, \ldots, s_{5}\right\}$, then (2) and $\varepsilon<t_{1}$ imply $\gamma\left(F_{f}\right) \cdot z+\gamma_{0}\left(F_{f}\right)=\prod_{i=1}^{5}\left(\varepsilon-s_{i}\right)<0$, and

$$
\alpha\left(F_{f}\right) \cdot v=\gamma\left(F_{f}\right) \cdot v=\gamma\left(F_{f}\right) \cdot z-N<-\gamma_{0}\left(F_{f}\right)-N<-\gamma_{0}\left(F_{f}\right)=\alpha_{0}\left(F_{f}\right) .
$$

- If $0 \in f$, then $\gamma_{0}\left(F_{f}\right)=0$ and $f=\{0,1\} \cup\{i, i+1\} \cup\{n\}$ with $2 \leq i \leq n-2$. By inequality (52) and the definition of $\prec$, we conclude that necessarily $\gamma\left(F_{f}\right) \cdot z<N$ and

$$
\alpha\left(F_{f}\right) \cdot v=\gamma\left(F_{f}\right) \cdot z-N<0=\alpha_{0}\left(F_{f}\right) .
$$

We have verified the inequalities (S4) and completed the proof of Theorem 2 .

Remark 2. A referee has suggested to extend this construction to boundaries of more general even-dimensional squeezed balls. However, so far we have only been able to realize odd-dimensional squeezed spheres directly modeled on the 3 -dimensional ones, and leave this as an open problem.

\section{A shorter proof that squeezed 3-spheres are Hamiltonian}

In 1973, BARNETTE [8] conjectured that all simple 4-polytopes admit a Hamiltonian circuit. In [4], HeBBLE and LEE prove that squeezed 3-spheres are (dual) Hamiltonian by explicitly constructing a Hamiltonian circuit in the dual graph; however, their proof goes through extensive case analysis. A referee has suggested that it might be possible to obtain a simpler proof of this result. In this section, we follow his or her suggestion and obtain a "proof by picture" with fewer case distinctions, which moreover only depend on parity conditions. 
Theorem 3. (HeBBlE and LeE, 2000 四) The dual graph of any Kalai 4-polytope S(I) admits a Hamiltonian circuit. In particular, the polars of these 4-polytopes satisfy BARNETTE's conjecture.

Proof. Recall from Section 3.2 that the set of facets of $S(I)$ is $B\left(I^{\prime}\right) \cup\left(0 * S\left(I^{\prime}\right)\right)$. We continue to write $(i, j)=\{i, i+1, j, j+1\}$ for facets of $S(I)$ in $B\left(I^{\prime}\right)$, and introduce the notation $\left(i+\frac{1}{2}, j\right):=$ $\{0, i+1, j, j+1\}$ and $\left(i, j+\frac{1}{2}\right):=\{0, i, i+1, j+1\}$ for facets of $S(I)$ in $0 * S\left(I^{\prime}\right)$. Also, recall from Section 3.2 the definition of the order relations $\preceq$ and $\sqsubset$, and number the set $G^{\prime}$ of $\preceq$-maximal elements $\left(i_{k}, j_{k}\right)$ of $B\left(I^{\prime}\right)$ in ascending $\sqsubset$-order, starting with $k=1$.

We start our Hamiltonian circuit in the dual graph of $S(I)$ at the facet $\left(i_{0}, j_{0}\right)=(1,3)=$ $\{1,2,3,4\} \in B\left(I^{\prime}\right)$. While walking through the other facets of $B\left(I^{\prime}\right)$, we will also pick up the facets of the form $\left(i+\frac{1}{2}, j\right)$ and $\left(i, j+\frac{1}{2}\right)$ with $i, j \geq 1$ of $S\left(I^{\prime}\right)$, and then return to $(1,3)$ via the set of facets $\{(0, j): 2 \leq j \leq n-1\}$. We will also use the difference operators $\Delta j_{k}=j_{k+1}-j_{k}$ and $\Delta i_{k}=i_{k+1}-i_{k}$. In our circuit, we repeatedly go through certain steps, and in the figures we will mark the end of one step and the beginning of the next by a square. In all steps, if all facets in $G^{\prime}$ are processed, go to step Down (and then to Finish).

1. Over the top: Start at $\left(i_{0}, j_{0}\right)=(1,3)$. If $j_{1}-j_{0}$ is odd, continue as in Figure 2(a). If $j_{1}-j_{0}$ is even, proceed as in Figure 2(b). In both cases, go on until $\left(i_{1}+\frac{1}{2}, j_{1}\right)$. Set $k=1$, and go to step Down.

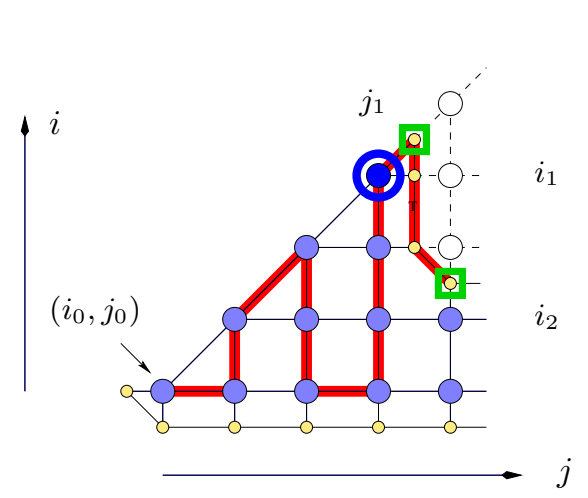

(a) $j_{1}-j_{0}$ odd

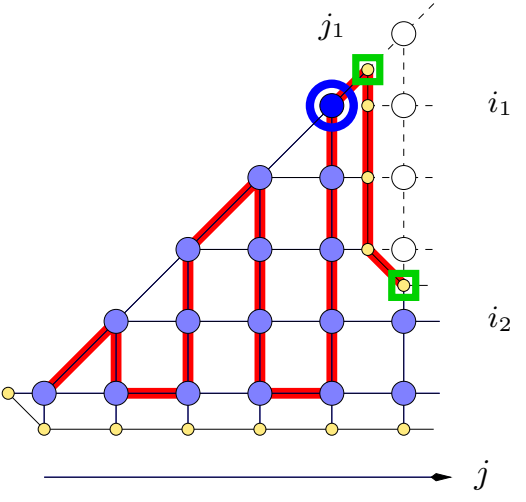

(b) $j_{1}-j_{0}$ even

Figure 2: Steps Over the top and Down. The circled facet is $\left(i_{1}, j_{1}\right)$, the upper $\square$ represents $\left(i_{1}+\frac{1}{2}, j_{1}\right)$, and the lower $\square$ is $\left(i_{2}+\frac{1}{2}, j_{1}+1\right)$.

2. Down: If there are no more generators to be processed, go down along the facets $\left\{\left(i_{\ell}, j_{k}+\frac{1}{2}\right)\right.$ : $\ell=k, k-1, \ldots, 1\}$ and continue with step Finish. Otherwise, if $\Delta i_{k}>0$, continue downwards as in Figure 2 until $\left(i_{k+1}+\frac{1}{2}, j_{k}+1\right)$. If $i_{k+1}=i_{k}$, do nothing. In both cases, increment $k$ by 1 , and continue to step Across.

3. Across: If $\Delta j_{k}$ is even, continue as in Figure 3(a). If $\Delta j_{k}$ is odd and not 1 and $i_{k+1}-i_{0}$ is even, continue as in Figure 3(b); if $\Delta j_{k} \neq 1$ and $i_{k+1}-i_{0}$ are both odd, as in Figure 3(c).

If $\Delta j_{k}=1$ and $\Delta i_{k+1}$ is even, proceed as in Figure $4(\mathrm{a})$, if $\Delta i_{k+1}$ is odd, as in Figure $4(\mathrm{~b})$. In any case, increment $k$ by one, and repeat from step Down or Across as necessary, depending on whether the facet surrounded by a dashed circle in Figure 1 is in $G$ or not.

4. Finish: Now the only thing left to do is to return to $(1,3)$ via the set of facets $\{(0, j)$ : $n-1 \geq j \geq 2\}$, as in Figure 5 .

This completes the proof of Theorem 3 . 


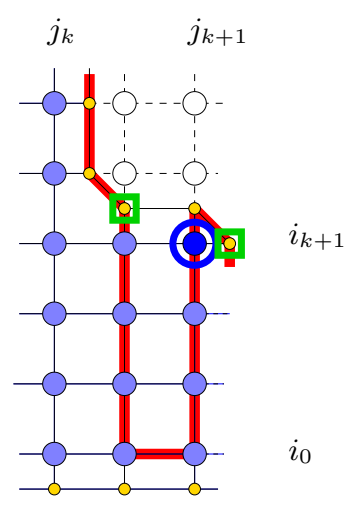

(a) $\Delta j_{k}$ even

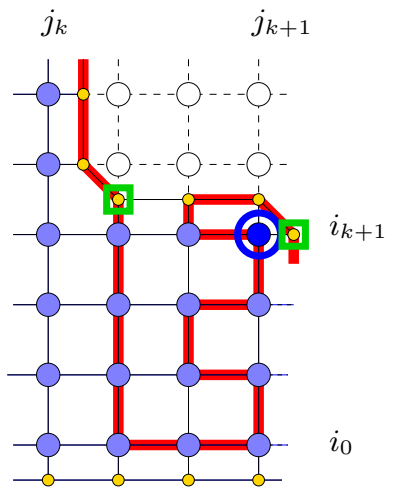

(b) $\Delta j_{k}$ odd, $i_{k+1}-i_{0}$ odd

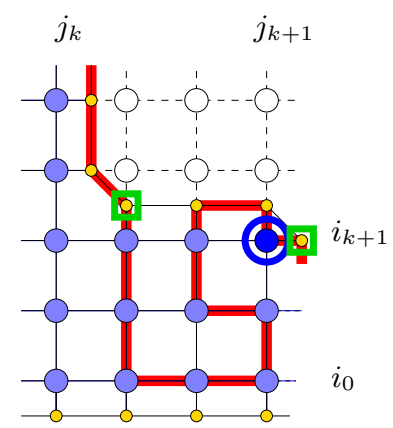

(c) $\Delta j_{k}$ odd, $i_{k+1}-i_{0}$ even

Figure 3: Step Across in case $\Delta j_{k}$ is even. The circled facet is $\left(i_{k+1}, j_{k+1}\right)$.

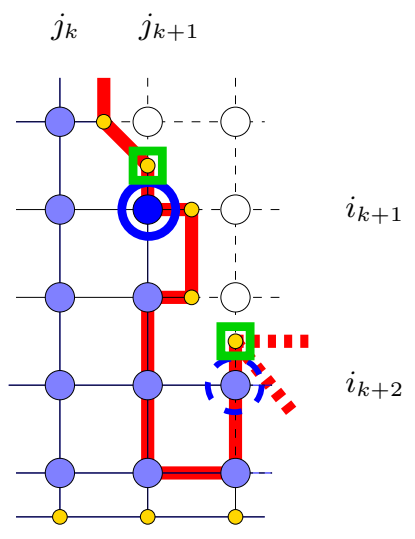

(a) $\Delta i_{k+1}$ even

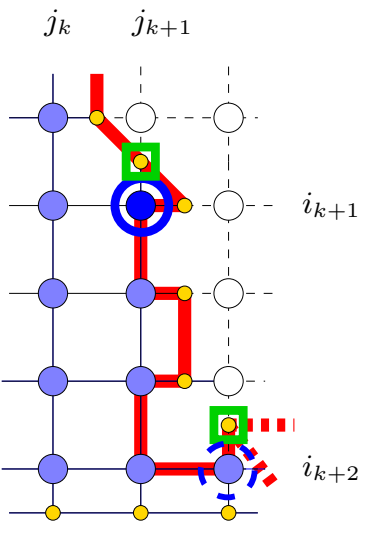

(b) $\Delta i_{k+1}$ odd

Figure 4: Step Across in case $\Delta j_{k}=1$. The circled facet is $\left(i_{k+1}, j_{k+1}\right)$. Depending on whether the facet surrounded by a dashed circle in Figure $⿴$ is in $G$ or not, the next step will be Down or Across, respectively.

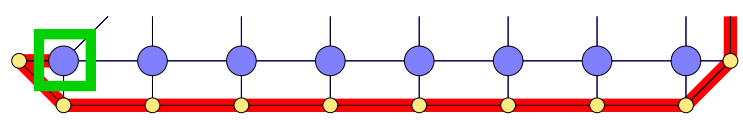

Figure 5: Step Finish. 


\section{Acknowledgements}

It is a great pleasure to thank GüNTER M. ZIEGLER for suggesting this problem, his many helpful comments, and his patience in going through various versions of this paper. Special thanks also to Volker KaIBel for his very careful reading, to GüNTER Rote for pointing out a gap in my

first version of the realization construction, and to all members of the Discrete Geometry group at TU Berlin for their great support and the wonderful working environment there.

\section{References}

[1] L. Billera and C. W. Lee, A proof of the sufficiency of McMullen's conditions for $f$-vectors of simplicial convex polytopes, J. Combin. Theory, Ser. A 31 (1981), pp. 237-255.

[2] J. E. Goodman and R. Pollack, There are asymptotically far fewer polytopes than we thought, Bull. Amer. Math. Soc. 14 (1986), pp. 127-129.

[3] J. E. Goodman and R. Pollack, Upper bounds for configurations and polytopes in $\mathbb{R}^{d}$, Discrete Comput. Geom. 1 (1986), pp. 219-227.

[4] R. L. Hebble and C. Lee, Squeezed 2-spheres and 3-spheres are Hamiltonian, preprint December 2000, 15 pages. http://www.ms.uky.edu/ ${ }^{2}$ lee/ham.pdf

[5] G. Kalai, Many triangulated spheres, Discrete Comput. Geom. 3 (1988), pp. 1-14.

[6] C. W. Lee, Kalai's squeezed spheres are shellable, Discrete Comput. Geom. 24 (The Branko Grünbaum Birthday Issue, 2000), pp. 391-396.

[7] P. McMullen, The numbers of faces of simplicial polytopes, Israel J. Math. 9 (1971), pp. 559 570.

[8] M. Rosenfeld and D. Barnette, Hamiltonian circuits in certain prisms, Discrete Math. 5 (1973), pp. 389-394.

[9] R. P. Stanley, The number of faces of a simplicial convex polytope, Advances in Math. 35 (1980), pp. 236-238.

[10] R. P. Stanley, Enumerative Combinatorics, Vol. 1, Second ed., Cambridge Studies in Advanced Mathematics, vol. 49, Cambridge University Press, 1997.

[11] G. M. Ziegler, Lectures on Polytopes, Graduate Texts in Mathematics, Vol. 152, SpringerVerlag, New York, 1995. Revised edition 1998. 